*For correspondence: mark.alkema@umassmed.edu (MA); zhen@lunenfeld.ca (MZ); samuel@physics.harvard.edu (ADTS)

Present address: ${ }^{\dagger}$ Department of ${ }^{10}$ Brain and Cognitive Sciences, MIT, Cambridge, MA USA; ${ }^{\ddagger}$ Department 11 of Physics, Northeastern University, Boston, MA USA

\section{Corollary Discharge Promotes a Sustained Motor State in a Neural Circuit for Navigation}

$4 \mathrm{Ni} \mathrm{ji}^{1 \dagger}$, Vivek Venkatachalam ${ }^{1 \ddagger}$, Hillary Rodgers ${ }^{1,3}$, Wesley Hung ${ }^{2}$, Taizo Kawano $^{2}$, Christopher M. Clark ${ }^{3}$, Maria Lim ${ }^{2}$, Mark J. Alkema ${ }^{3 *}$, Mei Zhen ${ }^{2 *}$, Aravinthan D.T. Samuel $^{1 *}$

$7{ }^{1}$ Department of Physics and Center for Brain Science, Harvard University, 17 Oxford 8 Street, Cambridge, MA 02138 USA; ${ }^{2}$ Lunenfeld-Tanenbaum Research Institute, Mount Sinai Hospital, Toronto, ON, Canada M5G 1X5; and Departments of Molecular Genetics, and Physiology, University of Toronto, Toronto, ON, Canada M5S 1A8

\begin{abstract}
Animals exhibit behavioral and neural responses that persist on longer time scales than transient or fluctuating stimulus inputs. Here, we report that C. elegans uses corollary discharge to sustain motor responses during thermotactic navigation. By imaging circuit activity in behaving animals, we show that a principal postsynaptic partner of the AFD thermosensory neuron, the AIY interneuron, encodes both temperature and motor state information. By optogenetic and genetic manipulation of this circuit, we demonstrate that the motor state representation in AIY is a corollary discharge signal. RIM, an interneuron that is connected with premotor interneurons, is required for corollary discharge. Ablation of RIM eliminates the motor representation in AIY, allows thermosensory representations to reach downstream premotor interneurons, and reduces the animal's ability to sustain forward movements during thermotaxis. We propose that corollary discharge underlies a positive feedback mechanism to generate persistent neural activity and sustained behavioral patterns in a sensorimotor transformation.
\end{abstract}

\section{Introduction}

Behavioral states often persist over longer timescales than their initiating sensory stimuli (Bidaye et al., 2014; Hoopfer et al., 2015). For example, fish continue to fixate their gaze on light after the onset of darkness (Seung, 1996; Aksay et al., 2007). A brief aversive stimulus evokes prolonged escape responses in many species (Li et al., 2006; Herberholz et al., 2002). Lasting behavioral states require circuit mechanisms to turn a transient stimulus into persistent neuronal activity (Lee and Dan, 2012; Major and Tank, 2004). One circuit topology that can produce persistent neural activity is positive feedback (Seung, 1996). However, establishing causality between positive feedback, persistent neural activity, and sustained behavior states has been challenging because of the technical difficulties in experimentally dissecting neural activities across entire sensorimotor pathways.

The compact nervous system and optical accessibility of $C$. elegans make it possible to explore molecular and circuit mechanisms that underlie persistent neural activities and sustained behavioral states in intact animals (Gao et alo, 2015). C. elegans requires persistent motor states to navigate variable sensory environments. During locomotion, the animal alternates between sustained forward movements and short reversals. When navigating through a chemical or thermal gradient, 
C. elegans exhibits biased random walks, extending forward runs towards preferred environments (Pierce-Shimomura et al., 1999; Ryu and Samuel, 2002; Ino and Yoshida, 2009). C. elegans can also exhibit klinotaxis, the gradual steering of heading angles during forward movements towards preferred directions (Ward, 1973; Ino and Yoshida, 2009). C. elegans employs both biased random walk and head steering (Hedgecock and Russell, 1975; Mori and Ohshima, 1995; Luo et al., 2014a) to actively move up or down a temperature gradient towards preferred temperatures - positive or negative thermotaxis, respectively.

The C. elegans wiring diagram has a layered organization (White et al., 1986) (Figure 2B). Sensory neurons communicate with first layer interneurons. Second layer interneurons communicate with head motor neurons and premotor interneurons that regulate body undulation. AFD is the thermosensory neuron that mediates both positive and negative thermotaxis (Luo et al., 2014a; Hawk et al., 2018). Its principal chemical synaptic partner, AIY, is the first layer interneuron specifically required for positive thermotaxis. Inactivation or ablation of AIY causes animals to exhibit negative thermotaxis at all temperatures (Mori and Ohshima, 1995; Hobert et al., 1997).

AIY responds to temperature variations due to signaling from AFD (Clark et al., 2006; Narayan et alo, 2011; Hawk et al., 2018). Excitatory synapses from AFD to AlY reliably transmit AFD's activity pattern into scaled AIY dynamics (Narayan et al., 2011). AIY is postsynaptic to multiple sensory neurons, and is thought to play a role in navigation across different sensory modalities by controlling the duration of forward runs (Gray et al., 2005; Wakabayashi et al., 2004; Tsalik and Hobert, 2003). AIY has also been shown to regulate the speed and direction of locomotion (Li et al., 2014; Kocabas et allo, 2012).

We probed mechanisms by which AIY biases random walks during positive thermotaxis. Our calcium imaging of AIY in moving animals reveals that AIY encodes both temperature and motor information. Consistent with a previous report (Luo et al., 2014b), we show that AIY activity rises at the onset of forward runs and falls at the onset of reversals. But we further reveal that whether AIY encodes the thermosensory input from the AFD neuron depends on motor state. During forward runs, AIY activity follows AFD activity, rising during warming and falling during cooling. During reversals, AIY does not encode AFD thermosensory input. We demonstrate that the motor state representation in AIY reflects corollary discharge (CD), a copy of the motor command. Corollary discharge to AIY requires RIM, an interneuron that is connected with both the forward and reversal premotor circuit. In the absence of RIM, AIY encodes thermosensory input from AFD regardless of motor state. Moreover, weak thermosensory representations appeared in some premotor interneurons. At the behavioral level, absence of RIM causes positive thermotaxis to be disrupted by reduced ability to sustain forward movement up temperature gradients. These experimental results support a minimal phenomenological model where both warming and corollary discharge reinforce and sustain the forward motor state in a biased random walk. Therefore, in C. elegans, motor state shapes sensory processing by feedback from premotor interneurons to first layer interneurons. Our results establish a role for corollary discharge in sustaining a motor state despite variable or fluctuating sensory environments.

\section{Results}

\section{Sustained forward movements across thermal fluctuations in positive thermotaxis}

C. elegans navigates towards temperatures that correspond to prior thermal experience. To evoke positive thermotaxis, we placed young adults cultivated at $25^{\circ} \mathrm{C}$ on a linear thermal gradient spanning 19 to $23^{\circ} \mathrm{C}$ (Figure 1). Consistent with earlier reports, these animals exhibited biased random walk and klinotaxis towards warmer temperatures (Figure 1B) ((Luo et al., 2014a; Yamaguchi et al., 2018)): runs that pointed in favorable directions were lengthened (Figure 1B; forward heading angles gradually reoriented towards temperatures that correspond to prior experience (Figure 1B). Without a temperature gradient, there was no evident modulation of either run length or heading angle (Figure 1B). 
Individual trajectories during positive thermotaxis revealed periods of forward movement that carry the animal up the temperature gradient. Although these periods of forward movement are persistent in duration, they are not always persistent in direction (Figure 1C). C. elegans experiences temporal changes in temperature on spatial gradients because of its own movements. Because of changes in movement direction, most runs - even those that orient the animal towards warmer temperatures - will typically involve detection of both warming and cooling stimuli (Figure 1D). Thus, C. elegans has an ability to sustain forward movement up temperature gradients across transient cooling fluctuations.

\section{Thermosensory encoding in AIY depends on motor state}

We sought circuit mechanisms for sustaining forward movement up temperature gradients despite thermal fluctuations.

First, we measured the activity of the AFD thermosensory neuron and AIY, its principle postsynaptic partner, by calcium imaging in moving animals. Subjected to oscillating temperatures below the preferred temperature, C. elegans exhibits positive thermotaxis. As previously reported (Clark et al., 2006, 2007), AFD's activity phase locks to periodic variations in temperatures, rising upon warming and falling upon cooling (Figure 2A). The power spectrum of AFD activity shows a strong peak corresponding to the frequency of thermal oscillation (Figure 2A). AFD activity showed no strong correlation with the motor state (Figure 2) (Spearman's $\rho<0.1, N=6$ ), indicating that motor commands arise downstream of the thermosensory neuron.

To understand the activity patterns of AIY, we simultaneously monitored AIY calcium dynamics along with components of the motor circuit known to code forward and reversal motor states (Figure 2B,C). We found that AIY encodes both motor and temperature information. During forward movements, AlY's calcium activity phase locked to temperature changes, increasing upon warming and decreasing upon cooling (Figure 2D). This response was attenuated during reversals (Figure 2D). As a result, the frequency of temperature oscillations is less well represented in the power spectrum of AlY activity than that of AFD ( $<<0.01$, Wilcoxon rank-sum test; $N=6$ ) (Figure 2D).

Unlike AFD and AIY, all motor circuit neurons that we examined strictly encode motor information during thermosensory stimulation. The AVA premotor interneuron is active during and regulates backward movement (Chalfie et al., 1985; Kawano et al., 2011; McCormick et al., 2011; Kato et al., 2015) (Figure 2B). In animals subjected to oscillating thermosensory stimulation, AVA calcium activity exhibited high and low states that correlated with backward and forward movement, respectively (Figure 2D). We detected no representation of the stimulus frequency in the power spectrum of AVA's activity pattern ( $p>0.1$, Wilcoxon rank-sum test, $N=5$, Figure $2 D$ ). Similarly, during thermosensory stimulation, the RME and SMD head motor neurons exhibited both high and low states that coincided with animal's directional movements (Figure 2D), as reported in previous studies (Hendricks, 2012). The power spectra of RME and SMD activity patterns also revealed no representation of thermosensory input (Figure 2D).

For positive thermotaxis, the sensorimotor transformation progresses through three layers (Figure 2B): the first layer encodes only thermal stimuli; the first layer interneuron encodes both thermal stimuli and motor states; the premotor and motor layer encode only motor states.

\section{Motor coding in AIY is a corollary discharge signal that requires the RIM interneu- ron \\ Our finding that AlY encodes thermal information in a manner that depends on motor state suggests a critical role in sensorimotor transformations during positive thermotaxis. In animals exposed to either constant or oscillating temperatures, AIY activity consistently rises at the beginning of forward runs and decays at the onset of reversals (Figure 3). How does AIY, a first order interneuron, acquire a motor signal? \\ We explored the possibility that proprioception, elicited by movement itself, underlies the calcium response in AlY. We imaged the thermosensory circuit activity in immobilized animals}


subjected to constant temperature. As in moving animals, AlY's activity remained anti-correlated with neurons active during reversals (AVA) and correlated with neurons active during forward movement (RME and SMDD/V) (Figure 2-Figure Supplement 1). C. elegans movement is not required for AlY activity to reflect motor state, arguing against proprioception.

We also tested whether motor commands are generated by AIY and transmitted to the premotor and motor circuits. We imaged AIY's activity upon blocking its chemical synaptic transmission by AlY-specific expression of tetanus toxin (TeTx) (Figure 3B). Without chemical synaptic output, AIY activity remained strongly coupled to the motor state, implying that AIY must receive the motor state signal.

We asked whether AIY receives corollary discharge from neurons that encode the motor command. We imaged AIY activity in moving animals upon ablation of AIY's downstream interneurons and premotor interneurons (Figure 4A). For interneurons, we focused on AIB and RIM. AIB shares electrical synapses with RIM and the AFD thermosensory neuron (White et al., 1986). In the context of chemotaxis, AIB and RIM have been shown to regulate variability in the neuronal and behavioral response to olfactory inputs (Gordus et al., 2015). We also tested AVA and AVB, premotor interneurons that regulate reversal and forward movement, respectively. Ablations were performed by expressing flavoprotein miniSOG, which induces acute functional loss and neuronal death by photoactivation (Qi et al., 2012).

We found that ablating AIB did not remove the motor state representation in AIY (Figure 4B, D). Neither did the removal of the premotor interneurons AVA or AVB alone (Figure 4B). However, AIY lost its motor state representation when we ablated RIM, either by itself or in combination with other premotor interneurons (Figure 4B, C).

RIM activity has been shown to be correlated with the AVA premotor interneuron that promotes reversals and anti-correlated with the AVB premotor interneuron that promotes forward movement (Kawano et al., 2011). To further probe whether RIM is required for the motor state signal to appear in AIY, we optogenetically activated either AVA or AVB while simultaneously measuring AIY calcium activity in immobilized animals. Activation of AVA using the light-gated opsin chrimson (Klapoetke et al., 2014) triggered a decrease in AIY calcium levels. Activation of AVB triggered an increase in AIY calcium levels Figure 4E). When RIM was ablated, AIY calcium signals no longer responded to optogenetic activation of either AVA or AVB, suggesting that RIM is part of the CD pathway from the motor circuit to AIY. Without RIM, AIY activity no longer reflected or depended on the motor state, but the premotor interneurons AVA and head motor neurons RME and RMD continued to encode for the backward and forward movement, albeit with reduced bimodal activity (Figure 5A,B). Thus, RIM is not essential for generating motor commands, but is necessary to relay motor information to AIY, a first layer interneuron.

\section{RIM-mediated corollary discharge does not depend on chemical synaptic transmis- sion}

We sought synaptic mechanisms by which RIM may contribute to the CD pathway. RIM expresses VGLUT3/EAT-4, indicating the potential involvement of glutamatergic synaptic transmission. RIM also synthesizes tyramine (Serrano-Saiz et al., 2013), a monoamine neuromodulator (Alkema et al., 2005). We imaged AIY activity in loss-of-function mutants for glutamatergic signaling (VGLUT3/eat4), tyramine synthesis (TDC/tdc-1), vesicular monoamine transport (VMAT/cat-1) and peptidergic signaling (CAPS/Unc-31). We found that AIY activity remained coupled to motor state in all mutants, but the difference in AIY activity between the backward and forward states was less distinct in mutants defective for vesicular monoamine transport (VMAT/cat-1) or tyramine synthesis (TDC/tdc-1) (Figure $5 C, D)$. This effect was similar to when we blocked RIM neurotransmitter release by TeTx (Ptdc-1::TeTx) (Figure 5C,D).

Since perturbation of chemical synaptic transmission did not abolish motor-related activity in AIY, neuronal communication that is independent of classic chemical synaptic transmission plays roles in relying corollary discharge to AIY. As previously reported (Kawano et al., 2011; Kato et al., 
2015; Gordus et al., 2015), RIM activity is strongly correlated with the AVA premotor interneuron, higher during reversals and lower during forward movement. AIY, on the other hand, exhibits increased activity during forward movement (Figure 3). Therefore, the corollary discharge signal must undergo sign reversal when propagated from RIM to AIY. Our observations suggest that the joint representation of sensory and motor signals in AIY arises from separate sources: feedforward input from AFD and feedback from the motor circuit that is conveyed through RIM.

\section{A role for RIM-dependent corollary discharge during positive thermotaxis}

RIM plays a critical role in the motor state dependent modulation of AlY calcium activity. This prompted us to examine the effect of disrupting the CD signal on sensorimotor transformations in behaving animals. When RIM-ablated animals were subjected to oscillating temperatures, AIY activity was no longer coupled to the motor state, but instead reliably tracked temperature fluctuations during both forward and backward movements (Figure 6). The stronger representation of an oscillating temperature in AlY activity was evident in its power spectrum (Figure 6A, B; $p<0.001$ Wilcoxon rank sum test). When RIM was ablated, we were also able to detect the representation of thermosensory oscillations in the activity pattern of the AVA premotor interneuron and the SMDD head motor neuron (Figure 6). This observation suggests that the loss of the RIM-dependent CD signal resulted in a sensorimotor circuit that becomes more susceptible to fluctuations in thermosensory input. Without RIM and the CD signal to AIY, thermosensory representations of fluctuating inputs can reach the motor circuit. The CD signal may thus play an important role in sustaining motor outputs across rapidly varying sensory inputs.

We tested this hypothesis by examining the effect of RIM ablation on positive thermotaxis (Figure 6D). These animals were specifically defective in their ability to sustain forward locomotion when moving up the thermal gradient (Figure $6 \mathrm{E}$ ). The gradual heading angle reorientation during a forward run remained intact (Figure $6 \mathrm{~F}$ ). Thus, the thermotaxis defect of RIM ablated animals is a disruption in the ability to sustain forward runs up temperature gradients.

\section{Agent-based simulations driven by a reduced model recapitulate the role of CD feedback in positive thermotaxis \\ To illustrate how corollary discharge could contribute to sustained motor states during thermotaxis, we built a minimal phenomenological model of the thermotaxis circuit (Figure 7). In this model, temperature fluctuations encoded by a thermosensory neuron is conveyed to a downstream interneuron. The interneuron outputs to a motor command circuit that determines the motor state. A copy of the motor command is relayed back to the interneuron in a manner that reinforces the ongoing motor state, effectively forming a positive feedback loop. When exposed to fluctuating inputs, this circuit transitioned between two stable states at time scales much longer than the input signal (Figure 7B). \\ We used this circuit model to simulate animal locomotion along linear thermal gradients (Fig- ure $7 \mathrm{C}$ ). The model with strong corollary discharge most effectively drove migration up the tempera- ture gradient. In this case, forward runs up the temperature gradient were substantially longer than those down the gradient (Figure 7D,E). Decreasing the strength of corollary discharge lessened the dependence of run length on run direction, and led to less efficient and less reliable thermotaxis (Figure 7C, D, E).}

\section{Discussion}

Positive feedback as a circuit topology has been proposed to sustain neural activity patterns (Seung, 1996; Major and Tank, 2004). Here, we uncovered a role for corollary discharge, a feedback signal from the motor circuit, in sustaining a neural state for forward locomotion. By relaying a motor command to a first layer interneuron in the sensorimotor pathway, this circuitry couples warming signals with forward motor state signals. These signals reinforce each another, leading to sustained 
periods of forward movement up temperature gradients. Sustained neural activity states allow the animal to filter rapid fluctuations in sensory input from affecting motor behavior, thereby enabling persistent behavioral outputs.

Emerging evidence from across species indicates that motor states can significantly impact sensory processing (Seelig and Jayaraman, 2015; Fu et al., 2014; Schneider et al., 2014; Zagha et al., 2013; Petreanu et al., 2009; Ouellette et al., 2018). An explicit dependence of sensory encoding on behavioral states may contribute to observed variability in stimulus-evoked behavioral responses. In C. elegans chemotaxis, variability in neuronal and behavioral responses has been linked to the RIM interneuron. The AWC olfactory neuron reliably responds to olfactory inputs, but its downstream partner, the AIB interneuron, responds less reliably. Ablation of RIM reduces the variability in the AIB response. One interpretation of the role of RIM in the olfactory circuit is that it enhances the variability of a probabilistic sensorimotor transformation during the biased random walk towards chemoattractants (Gordus et al., 2015). By studying the circuit in moving animals, our results favor the interpretation for a different role for RIM. During thermotaxis, RIM allows AIY, a first-layer interneuron, to generate response variability that is not stochastic but directly correlated with motor state. This observation underscores the importance of measuring sensorimotor transformations in behaving animals where feedback loops are active.

The motor state signal in the AIY interneuron requires the RIM interneuron. We do not know the synaptic mechanism by which the corollary discharge reaches AlY. One possibility is that it employs synaptic transmission-independent mechanisms and/or indirect feedback circuits from RIM to AIY. More extensive molecular and cellular dissection is needed to understand how the corollary discharge signal reaches AIY (Figure 2). Ablation of RIM not only eliminates the motor state representation in AIY, but also leads to increased thermosensory representation in the activity patterns of downstream neurons. Thus, the positive feedback provided to the first layer interneuron contributes to the separation of sensory input patterns in sensory neurons from motor output patterns in premotor interneurons. Behaviorally, it enhances the stability of a motor state that carries the animal up temperature gradients during positive thermotaxis, an interpretation supported by agent-based modeling (Figure 7).

In conclusion, our findings reveal a new role for corollary discharge. In C. elegans thermotaxis, corollary discharge promotes sustained neural responses to thermal stimuli and stabilizes a motor state, enhancing the efficiency of thermotactic navigation.

\section{Methods and Materials}

\section{Molecular biology and transgenic strain construction}

Promoters

The following promoters were used to allow neuron-specific expression of a calcium sensor, chrimson, and miniSOG. Most were generated from genomic DNA isolated from mixed stage N2 animals. Promoters include $4.8 \mathrm{~kb}$ (Prig-3), 0.9 kb (Pinx-1), 5.3 kb (Pg/r-1), 2.9 kb (Pcex-1), 0.86 kb (Plgc-55B), $3.1 \mathrm{~kb}$ (Pnmr-1) genomic sequence. All promoters except Pnmr-1 and Plgc-55B used the genomic sequence of the respective length starting immediately upstream of the predicted ATG start codon of the respective genes. For Pnmr-1, a $2 \mathrm{~kb}$ internal fragment which reduces the $5.1 \mathrm{~kb} n m r-1$ reporter expression was removed (Kawano et al., 2011). Details on Plgc-55B can be found in (Gao et al., 2015).

\section{Calcium imaging}

For AlY calcium imaging, aeals003 was generated by integrating olaEx1621 [Pmod-1::GCaMP6s; Pttx3::RFP; Punc-122::mCherry]). The integrant was outcrossed against N2 for 4 times to generate strain ADS003, and crossed into lite-1 to generate QW1410.

For AFD calcium imaging, aeals004 was generated by integrating an existing Ex line [Pgcy8::GCaMP6s; Pgcy-8:::RFP; Punc-122:::mCherry]. The integrant was outcrossed against N2 for 4 times to generate strain ADS004. 
For premotor interneuron and motor neuron calcium imaging, pJH3338 was constructed for calcium imaging for premotor interneurons and head motor neurons. The GCaMP6s reporter was optimized for C. elegans and contained three C. elegans introns (Lim et al., 2016; Chen et al., 2013). GCaMP6s was fused with codon-optimized mCherry (wCherry) at the C-terminus to facilitate ratiometric measurement via simultaneous imaging of GFP and RFP. The reporter expression was driven by Pg/r-1 as described above. This construct was co-injected with lin-15(+) marker to lin$15(n 765)$ animals to generate extrachromosomal transgenic array $h p E x 3550$, and subsequently integrated to generate $h p / s 471$. The integrated array was outcrossed against N2 wild-type 4 times to generate ZM8558. For simultaneous AIY and premoter/interneuron imaging, $h p / s 471$ was crossed with aeals003 to generate ADS027.

\section{Neuron ablation}

pJH2829, pJH3311, pJH2931, pJH2890, and pJH2827 were constructed for LED-based neuronal ablation for RIM, AIB, AVA (plus other neurons), AVB (plus other neurons), and AVA/AVE/AVD/RIM/PVC (plus other neurons), respectively. miniSOG fused with an outer mitochondrial membrane tag TOMM20 (tomm20-miniSOG or mito-miniSOG) (Qi et al., 2012; Shu et al., 2011). An inter-cistronic sequence splice leader (SL2) was inserted between the coding sequence of tomm20-miniSOG and codon-optimized mCherry (wCherry; a gift of A. Desai, UCSD) to visualize neurons that express miniSOG, and to examine the efficacy of ablation. SL2 sequence was PCR amplified off the splice leader sequence (SL2) between gpd-2 and gpd-3. These constructs were co-injected with the lin-15(+) marker in lin-15(n765) animals to generate extrachromosomal arrays hpEx2997, hpEx3464, hpEx3072, $h p E x 3064$, and $h p E x 2940$, respectively. With the exception of $h p E x 3072$, other arrays were integrated to generate $h p / s 327, h p / s 465, h p / s 331$, and $h p / s 321$. All integrated transgenic arrays were outcrossed 4 times against N2, except hpls327, which was outcrossed 7 times against N2, before being used for behavioral analyses or to be combined with AIY calcium imaging analyses, or, behavioral analyses.

AIY imaging upon neuronal ablation

aeals003 was crossed with $h p / s 327, h p / s 321, h p E x 3072, h p / s 331$, and $h p / s 465$, respectively, to generate ADS010, ADS014, ADS026, ADS036 and ADS046. They were used for AlY calcium imaging upon ablation of RIM, premotor interneurons (with a few other neurons), and AIB, respectively.

AlY calcium imaging upon genetic manipulation of synaptic transmission and optogenetic stimulation

For AlY imaging in genetic synaptic transmission mutants, QW1408, QW1409, QW1411, QW1175, and QW1415 were generated by crossing aeals003 into the corresponding mutant backgrounds listed in Supplemental Table 1.

For AlY imaging upon cell-type specific manipulation of synaptic transmission, aeals003 was crossed with yxIs25, xuEx1414, and kyEx4962 to generate ADS043, ADS042, and ADS013, respectively (Li et al., 2014; Zhang et al., 2005; Gordus et al., 2015).

Chrimson (Klapoetke et al., 2014) was codon-optimized and fused at C-terminus with wCherry as described (Lim et al., 2016). Chrimson expression was driven by Plgc-55B and Prig-3 to generate pHR2 and pHR6. These constructs were co-injected with Pges-1::GFP into QW1410 to generate aeaEx003 (ADS29) and aeaEX005 (ADS31), for AIY imaging upon optogenetic stimulation of AVB and AVA, respectively.

aeaEx003 and aeaEx005 were then crossed into hpls327;aeals003; lite-1 to generate ADS033 and ADS035 for AIY calcium imaging in RIM ablated animals, upon AVB and AVA stimulation, respectively.

\section{Behavioral assays}

Positive thermotaxis assay

$\mathrm{L} 4$ animals were cultivated at $25^{\circ} \mathrm{C}$ the night before the assay. On the day of the experiment, the behavioral arena was allowed to equilibrate until a stable linear thermal gradient spanning $19^{\circ} \mathrm{C}$ to 
$23^{\circ} \mathrm{C}$ was established. Before each assay session, a thin layer of NGM agar sized $20 \mathrm{~cm}$ on each side was placed on the arena and allowed to equilibrate to the temperature of the arena. Twenty young adults were collected from their cultivation plates and briefly washed in NGM buffer before they were transferred onto the thin agar. These animals were allowed to explore the assay environment for 5 minutes before behavioral recording starts. Afterwards, a CMOS camera positioned above the arena recorded continuously every 500 milliseconds for 20 minutes. Animal trajectories were extracted from the raw behavioral recordings using custom-written LABVIEW software. Subsequent analyses were performed in MATLAB.

\section{Spontaneous locomotion assay}

Animals were cultivated and prepared for behavioral assay in identical manners as for the positive thermotaxis assay. The same behavioral arena, equilibrate to room temperature $\left(22^{\circ} \mathrm{C}\right)$, was used to assay spontaneous locomotion. Behavioral recordings were conducted the same way as in the positive thermotaxis assay. Subsequent analyses were performed using the same LABVIEW software as above and subsequently in MATLAB.

\section{Calculation of thermotactic bias}

For each animal, the instantaneous velocity $(v)$ and speed $(|v|)$ were calculated from the animal's centroid positions. The velocity vector was then projected onto direction of the thermal gradient, which in this case was parallel to the negative direction of the $x$-axis of the behavior arena. The thermotactic bias as the ratio between the velocity projection along the thermal gradient and the instantaneous speed of the animal:

$$
\text { thermotactic bias }=\frac{-v_{x}}{|v|}
$$

\section{Calcium imaging}

Sample preparation and imaging setup

L4 larval animals expressing cytosolic GCaMP6s::wCherry were cultivated at $25^{\circ} \mathrm{C}$ the night before the imaging experiment. Immediately before the imaging session, animals were transferred to a microscope slide with a 5\% agarose pad ( $2 \mathrm{~mm}$ thick). A small drop of NGM buffer was added to the agarose pad and a \# 1 coverslip was lowered onto the pad where animals could execute forward runs and reversals in a restricted area. Calcium imaging was performed on an upright spinning disc confocal microscope (Nikon Eclipse LV100 and Yokogawa CSU22) and iXon3 DU-897 EMCCD camera (Andor). High resolution images were collected through a 40x, 0.95 NA Nikon Plan Apo lambda objective. 3D volumetric stacks were acquired in both the green (GCaMP6s) and red (wCherry) channels with an exposure of $30 \mathrm{~ms}$ at approximately 1.2 volumes per second.

\section{Control of thermal stimulation}

Animals were imaged on a custom-built temperature control stage where a PID controller and H-bridge amplifier (Accuthermo) drove a thermoelectric cooler (TEC) (Newark) that pumped heat into and out of a thin copper plate with a liquid-cooled water block (Swiftech) acting as a thermal reservoir. A type-T thermocouple microprobe (Physitemp) was placed on the copper plate underneath a thin steel tab. A custom written Labview program was used to specify the desired temperature waveform.

\section{Extraction of calcium transient levels}

To extract fluorescence intensities for individual neurons, we identified connected regions above a predefined intensity threshold and registered these regions of interest across a movie based on spatial proximity across frames. The activity level of each neuron was defined by $\Delta R(t) / R_{0}$ where $R(t)$ is the ratio between the GCaMP6s intensity and the wCherry intensity at time point $t$, and $R_{0}$ is defined as the median of the lowest ten $R(t)$ values in the time series. 


\section{Optogenetic stimulation and simultaneous calcium imaging}

Experimental animals expressing Chrimson were grown on NGM plates supplied with $5 \mu \mathrm{M}$ all-trans retinal (ATR) mixed with OP50 bacteria. Control animals of the same genotypes were grown on NGM plates seeded with OP50 without ATR. The day before the experiment, L4 animals were picked onto fresh plates (with ATR for the experimental groups and without ATR for the control groups). On the day of the experiment, young adult animals were prepared for imaging in the semi-constrained preparation as described above. During imaging, pulses of red light were delivered from a filtered white LED lamp. Pulse timing was controlled by MATLAB scripts. For calcium imaging, animals were illuminated with only the blue laser ( $488 \mathrm{~nm}$ ) to avoid strong activation of Chrimson.

\section{Neuron ablation}

Transgenic animals expressing miniSOG were collected from late $L 1$ to $L 2$ stage onto a small NGM plate (3.5 cm diameter). The plate was placed under a blue LED spotlight (Mightex, peak wavelength $617 \mathrm{~nm}$ ) for 40 minutes. Following illumination, the animals were allowed to recover for overnight at $15^{\circ} \mathrm{C}$ to examine the disappearance of cells. All ablation was performed using animals that carried integrated miniSOG transgens, with the exception for AVA ablation. Ablation of AVA was carried out in animals that carried an extrachromsomal array for Prig-3-miniSOG-SL2-RFP, which was subjected to random loss during somatic division. Animals used for ablation were selected for those that did not show expression (hence ablation) in a pharyngeal neuron that affects the survival of ablated animals.

\section{Statistical analysis}

\section{Statistical tests}

The Wilcoxon rank-sum test were used in the following comparisons: 1) comparing calcium activity upon the initiation of forward runs or reversals between wild type animals and various neuronablation experiments, 2) comparing the probability of change in AIY activity upon the initiation of forward run or reversals between wild type and AIY::TeTX animals, 3) comparing the thermotactic bias between wild type and RIM ablated animals. To control for multiple comparison, $\mathrm{p}$ values were adjusted using the Benjamini-Hochberg correction. 95\% confidence intervals were determined by bootstrapping.

Power spectral density for each calcium activity time series was estimated by first subtracting the mean of the time series, then applying Fourier transform using the fft function in MATLAB, and taking the square of the resulting values.

The bimodality coefficient (BC) was calculated as:

$$
\mathrm{BC}=\frac{m_{3}^{2}+1}{m_{4}+3 \times \frac{(n-1)^{2}}{(n-2)(n-3)}}
$$

where $m_{3}$ is the skewness of the distribution, $m_{4}$ is the excess kurtosis, and $n$ the sample size. BC >0.555 is typically taken to indicate bimodality (Pfister et al., 2013).

\section{Modeling of circuit activity and behavior}

Neural circuit model

We use a reduced model to capture the interaction between the three key components of the thermotaxis circuit: $V_{1}(t)$, the activity of the AFD thermosensory neuron; $V_{2}(t)$, the activity of the AIY interneuron; $V_{3}(t)$, motor circuit activity. A leaky integrator model that captures the dynamics of these interconnected circuits (Figure $7 \mathrm{~A}$ ) is given by a set of coupled equations:

$$
\begin{gathered}
\tau_{1} \frac{d V_{1}}{d t}=-g_{L 1}\left(V_{1}-V_{L 1}\right)-V_{\text {stim }} \\
\tau_{2} \frac{d V_{2}}{d t}=-g_{L 2}\left(V_{2}-V_{L 2}\right)+F_{21} V_{1}+F_{23} V_{3}
\end{gathered}
$$




$$
\tau_{3} \frac{d V_{3}}{d t}=-g_{L 3}\left(V_{3}-V_{L 3}\right)+F_{32} V_{2}
$$

where $g_{L 1}, g_{L 2}$, and $g_{L 3}$ denote non-negative leak conductances and $V_{L 1}, V_{L 2}$, and $V_{L 3}$ denote resting potentials. Synaptic interactions between neurons are modeled as sigmoidal functions, based on measurements in a related nematode species (Ferrée et al., 1999):

$$
\begin{aligned}
& F_{21}\left(V_{1}\right)=-\overline{g_{1}}\left(\frac{1}{1+\exp -k_{1} *\left(V_{1}-\beta_{1}\right)}\right) \\
& F_{23}\left(V_{3}\right)=-\overline{g_{2}}\left(\frac{1}{1+\exp -k_{2} *\left(V_{3}-\beta_{3}\right)}\right) \\
& F_{32}\left(V_{2}\right)=-\overline{g_{3}}\left(\frac{1}{1+\exp -k_{3} *\left(V_{2}-\beta_{2}\right)}\right)
\end{aligned}
$$

where $g_{i}, k_{i}, \beta_{i}$ define the height, steepness and inflection point of each sigmoidal function.

Previous studies have shown that, at temperatures close to the prior cultivation temperature, AFD activity reliably reports the temporal derivative of the ambient temperature. To simplify the model and focus on the role of positive feedback in the circuit, we approximated AFD activity by its steady state value:

$$
V_{1}(t) \cong V_{1 \infty}=V_{L 1}+\frac{V_{\text {stim }}}{g_{L 1}}=V_{L 1}+\alpha \frac{d T}{d t}
$$

Substituting Equation 7 into Equation 2 reduces the model to two dimensions:

$$
\begin{gathered}
\tau_{2} \frac{d V_{2}}{d t}=-g_{L 2} V_{2}+\alpha \frac{d T}{d t}+F_{23}\left(V_{3}\right)+C_{2} \\
\tau_{M} \frac{d V_{3}}{d t}=-g_{L 3} V_{3}+F_{32}\left(V_{2}\right)+C_{3}
\end{gathered}
$$

where $C_{2}=g_{L 2} V_{L 2}+V_{L 1}$ and $C_{3}=g_{L 3} V_{L 3}$.

Numerical integration of the governing equations was used to generate the simulations shown in Figure 7.

\section{Simulation of thermotaxis behavior}

To simulate behavior, we model the locomotory state of the animal, $S$, as a function of the net activity of the motor circuit:

$$
S(t)=\operatorname{sgn}\left(V_{3}(t)\right)
$$

where $S(t) \geq 0$ is defined as the forward run state and $S(t)<0$ is defined as the reversal state.

During an ongoing forward run or reversal, the heading direction, $\theta(t)$, remains constant. At the start of each forward run, the new heading direction is chosen randomly from a uniform distribution with range $0- \pm 180^{\circ}$. When a forward run ends and a reversal state starts, the heading direction changes by $180^{\circ}$ :

$$
\theta(t)= \begin{cases}\left(\cos \theta_{0}, \sin \theta_{0}\right) & t=0 \\ \theta(t-d t) & S(t)=S(t-d t) \\ -\theta(t-d t) & S(t)=1 \text { and } S(t-d t)=-1 \\ \left.\cos \theta_{0}, \sin \theta_{0}\right) & S(t)=-1 \text { and } S(t-d t)=1\end{cases}
$$

To isolate the effect of corollary discharge on the duration of behavioral states, all animals are simulated to move at constant speed (1 unit length per time step).

The stimulus environment is also chosen to simulate experimental conditions. A linear thermal gradient along the $x$ direction is set by $T(x)=c_{T} x$. 


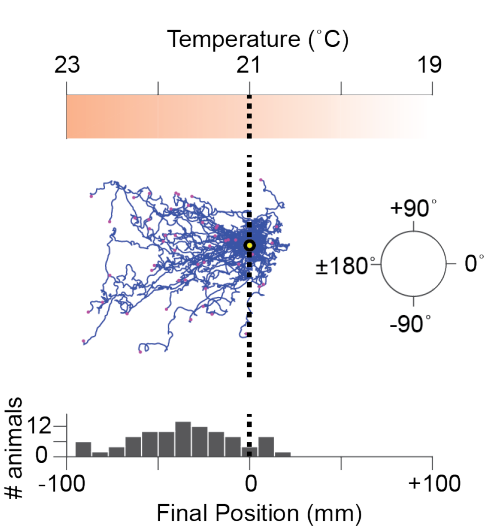

C

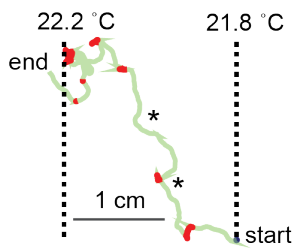

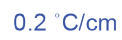

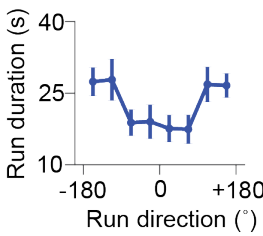

no gradient
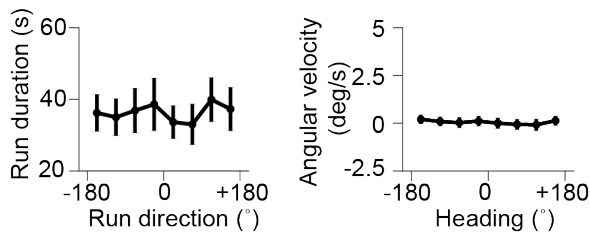

D

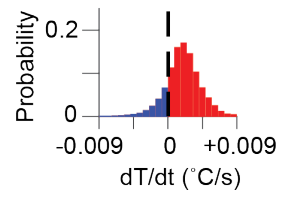

Figure 1. Sustained forward motor state despite of temperature fluctuations during positive thermotaxis. A. Example trajectories of wild type C. elegans cultivated at $25^{\circ} \mathrm{C}$ migrating up a linear temperature gradient over a 20-minute period. Top, schematics of the thermal gradient. Middle, trajectories of 49 animals during positive thermotaxis. The starting points of all trajectories are aligned (yellow dot) and the end points are marked by magenta dots. Bottom, a histogram of the final location of animals. B. Quantification of the duration of forward run and the angular velocity of run direction as a function of direction of forward run on spatial thermal gradients (top, $n=140$ ) and on constant temperature surfaces (bottom, $n=140$ ). Error bars are $95 \%$ confidence intervals $(95 \% \mathrm{Cl})$. C. Thermotaxis trajectory of a single animal during thermotaxis with alternating periods of forward movement and reversals (left), and the instantaneous heading angle over time during one extended period of forward movement within the trajectory (right). Asterisks denote periods where the heading direction pointed down the thermal gradient. D. Histogram of temporal changes in temperature $(d T / d t)$ experienced by animals during forward runs that ended up pointing up the temperature gradient $(n=164)$.

The instantaneous temperature, $T$, experienced by the animal is a function of the animal's position on the thermal gradient, $T(t)=c_{T} P_{x}(t)$ where $\vec{P}=\int_{t_{0}}^{t} \vec{v} d \tau$.

Each simulation is initialized by setting an starting position of $(0,0)$, an initial heading angle drawn from the uniform distribution from $0- \pm 180^{\circ}$, and by setting the animal in a forward run state, $V_{2}\left(t_{0}\right)=1$ and $V_{3}\left(t_{0}\right)=1$. Upon numerical integration, simulated worms move autonomously in their environment for a predetermined duration $\left(t_{\max }\right)$.

In the behavior simulations used to generate the data shown in Figure 7, we have assumed that the animal's direction of locomotion does not change during the forward run. This simplification is motivated by the observation that RIM ablation specifically disrupts the modulation of run duration, while leaving the modulation of run direction intact. Since the latter is likely controlled by neural circuitry not modeled here, we chose to focus on the modulation of run duration during thermotaxis. 
A
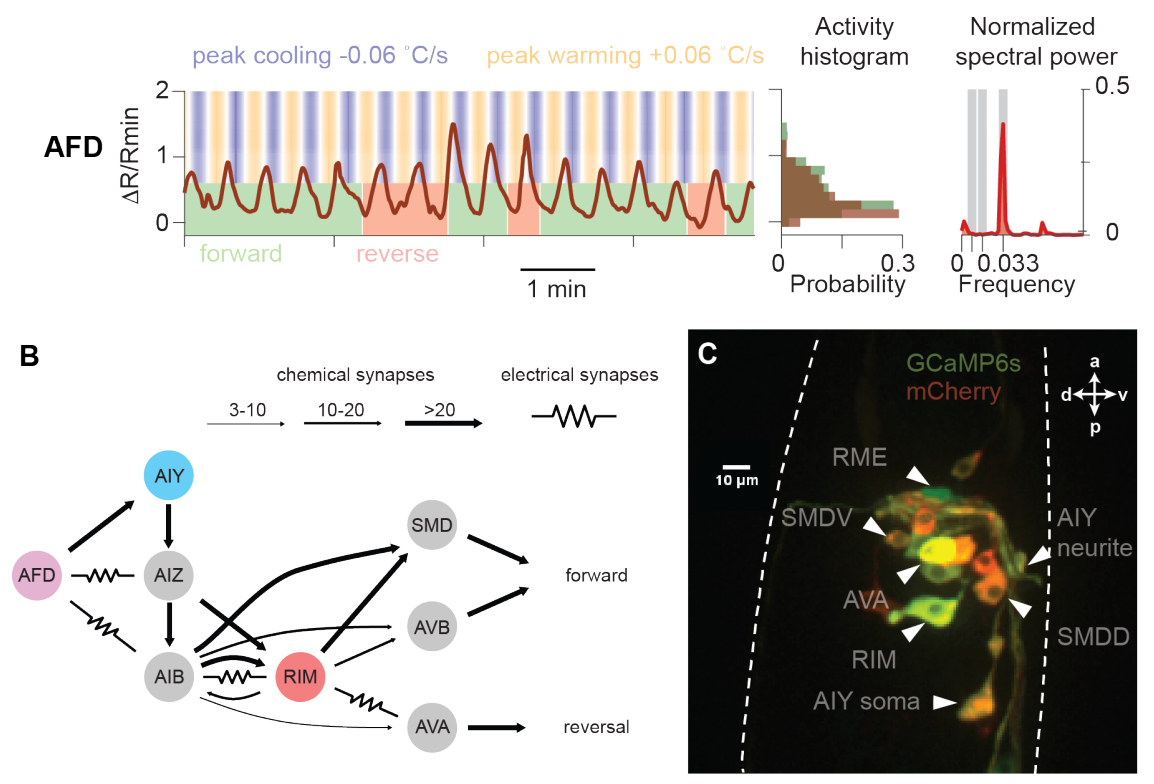

D

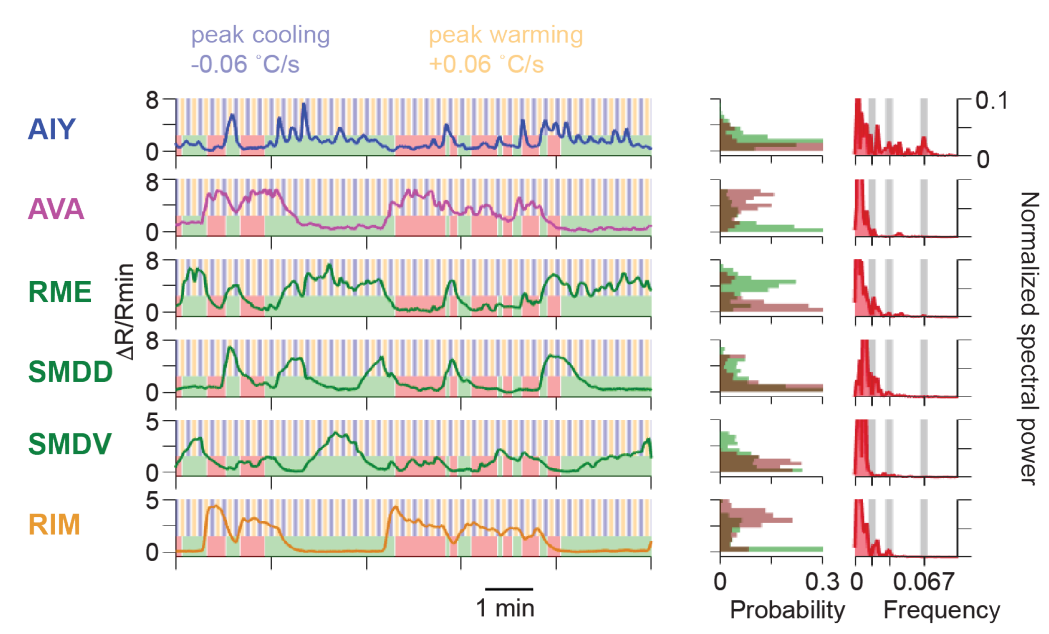

Figure 2. Neural activity patterns in the thermotactic circuit. A. An example trace of calcium transient of the AFD thermosensory neuron in moving wild type animals in response to an oscillating periodic thermal stimulus at a frequency of $f_{s}$ tim $=1 / 30 s^{-1}$. Calcium dynamics overlaid onto the temperature profile and motor state is shown to the left. The histogram and power spectrum of this example trace is shown to the right. Gray bands denote putative oscillation frequencies if the activity of AFD encoded perfectly $\left(f_{\text {response }}=f_{\text {stim }}\right.$ ) or imperfectly ( $f_{\text {response }}=1 / 2 * f_{\text {stim }}, 1 / 4 * f_{\text {stim }}$, etc.) the stimulus frequency. B. Anatomical connections between AFD, AIY, RIM, and key premotor and motor neurons involved in controlling locomotion during thermotaxis. C. Anatomic organziation of interneurons and motor neurons implicated in thermotaxis and/or locomotion, denoted by a transgenic strain that expresses the GCaMP6s::wCherry calcium reporter. D. Simultaneous measurement of calcium transient changes of neurons downstream of AFD in moving animals responding to a periodic thermal stimulus.

Figure 2-Figure supplement 1. Analysis of neural activity in the thermotaxis circuit in immobilized and moving animals without temperature stimulation. 
A

\author{
AIY::GCaMP6 \\ constant T
}

$\Delta \mathrm{R} / \mathrm{Rmin}$

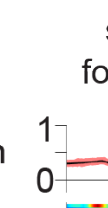

B

oscillating $T$

$\Delta R / R \min$

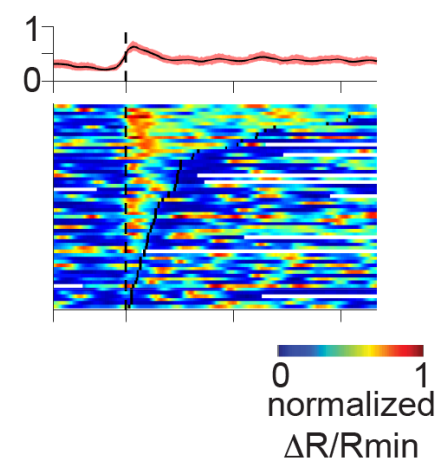

AlY::GCaMP6;
AlY::TeTX

start

$\Delta R / R \min$

$$
\text { forward }
$$

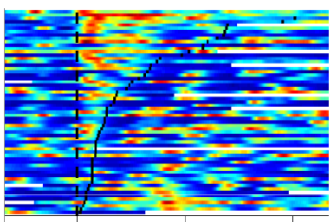

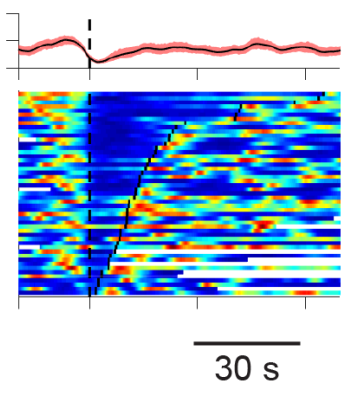

start reversal

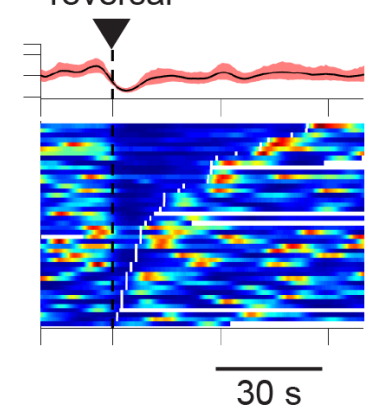

Figure 3. Motor-related activity in the AIY interneuron represents a CD signal.

A. Traces of calcium transients of the AIY interneuron aligned to the onset of forward runs (left column) or reversals (right column) in animals exposed to oscillating (top row, $\mathrm{N}=6$ animals) or constant (bottom row, $\mathrm{N}=5$ animals) temperature. Each row of the heat plots represents changes in AlY calcium transients a single behavioral epoch. The curve on top of each panel represents activity dynamics averaged across individual epochs. Broken black lines denote the onset and offset of individual behavioral epochs. B. AlY activity during forward runs and reversals in animals expressing tetanus toxin (TeTx) specifically in AlY at constant temperature ( $\mathrm{N}=4$ animals). 
A

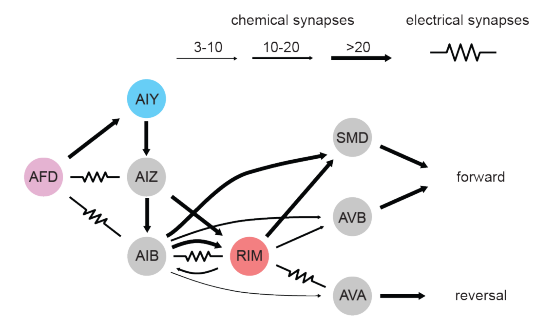

C

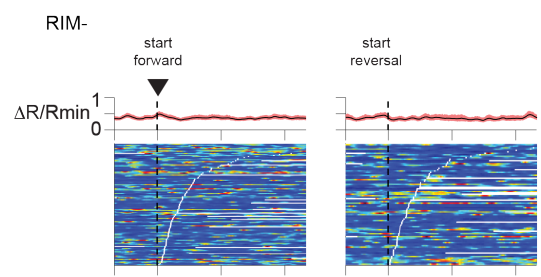

D

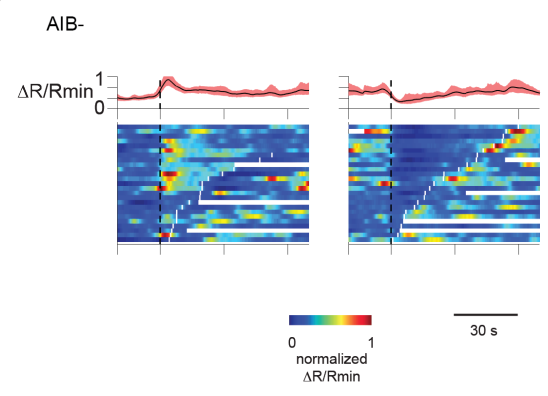

B

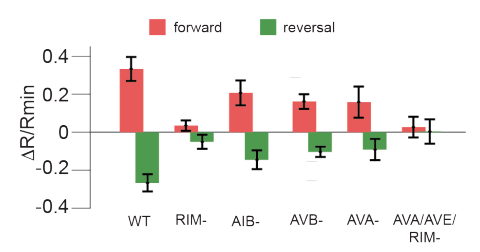

E

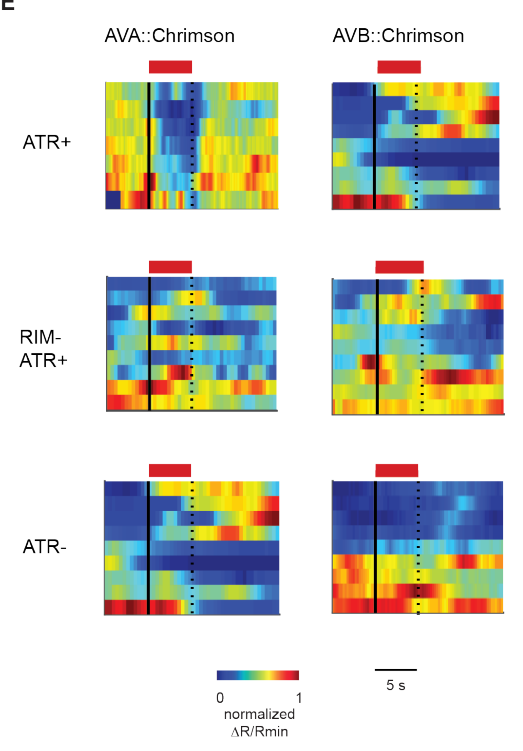

Figure 4. A CD pathway through the RIM interneuron couples AIY activity with the motor state.

A. Anatomical connections between AFD, AIY, RIM and key premotor and motor neurons involved in controlling locomotion during thermotaxis. B. Quantification of motor-related activity in AIY in animals carrying ablations in RIM, AIB, and premotor interneurons. C. AIY activity aligned to the onset of forward runs (left) or reversals (right) in animals where RIM has been genetically ablated ( $N$ $=8$ animals). Each row of the heat maps represent neural activity throughout a single behavioral epoch. The traces on top of the heat plots represent neural activity averaged across individual behavioral epochs. The broken lack lines denote the onset and offset of individual behavioral epochs. D. AIY activity aligned to the onset of forward runs (left) or reversals (right) in animals where the AIB interneurons have been genetically ablated ( $N=5$ animals). E. AIY activity in response to optogenetic stimulation of the AVA (left) or AVB (right) premotor interneurons (and others) in wild type animals grown on all-trans retinal (ATR) (top; $N=2$ animals for AVA, $N=5$ animals for AVB), RIM ablated animals grown on ATR (middle; $N=5$ animals for AVA, $N=6$ animals for AVB), and wild type animals grown without ATR (bottom, $N=5$ animals for AVA, $N=3$ animals for AVB). 
A

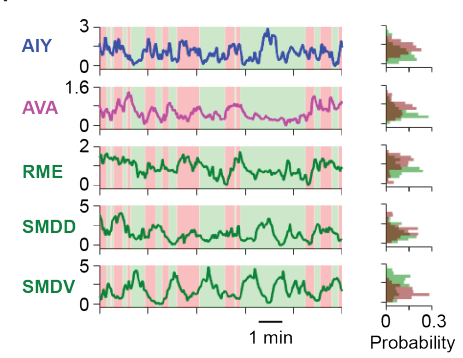

D

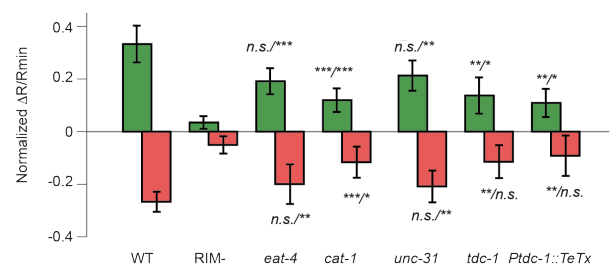

B
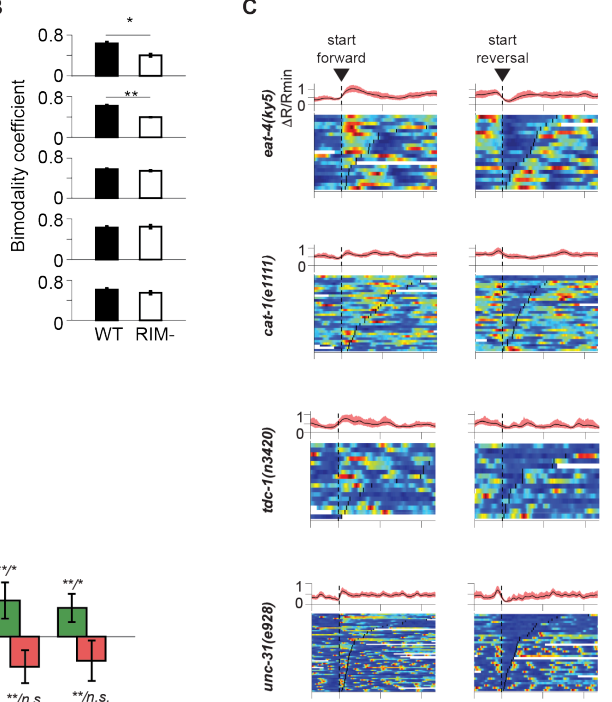

:

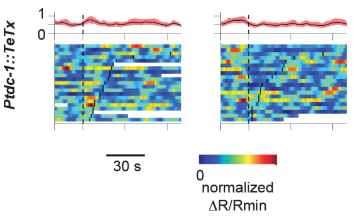

Figure 5. Loss of RIM eliminates motor state encoding in AIY. A. Simultaneous measurement of calcuim transient changes in AIY and neurons of the motor circuit in RIM ablated animals under constant temperature. Sample traces are shown to the left. Activity distribution for each neuron is shown to the right. B. Comparison of bi modality coefficients for the activity of individual neurons in wild type versus RIM ablated animals. C. AIY activity in VGLUT3/eat-4(ky5) mutants ( $\mathrm{N}=3$ animals); VAMT/cat-1(e1111) mutants ( $\mathrm{N}=3$ animals); CAPS/unc 31(e69) mutants ( $\mathrm{N}=3$ animals); TDC/tdc-1(n3420) mutants ( $\mathrm{N}=4$ animals); and in the transgenic animals expressing TeTx (tetanus toxin) specifically in the RIM and RIC neurons ( $N=4$ animals). $D$. Quantification of motor state activity in AIY in mutants and transgenic animals in (C). Significance of difference in mean between wild type (left) and RIM ablated (right) animals are presented on top of each bar. Error bars are $95 \%$ CI. n.s., non-significant, *, $p<0.05, * *, p<0.01, * * *, p<0.001$ by Wilcoxon rank-sum test. 
A
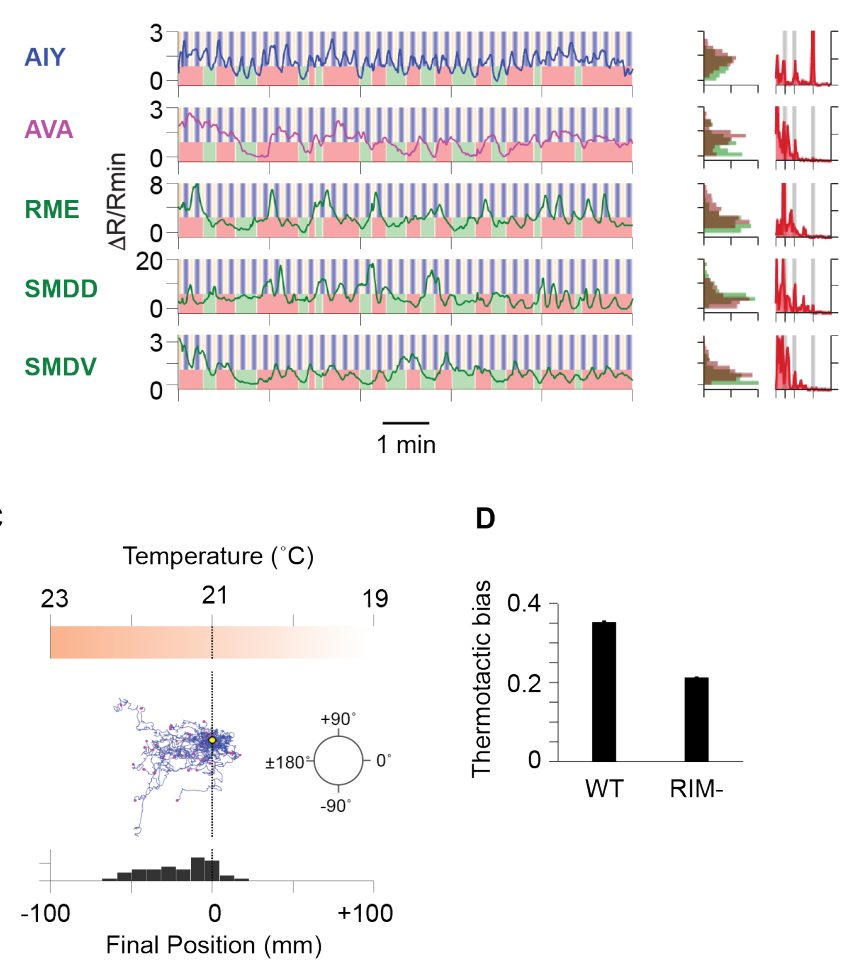

E

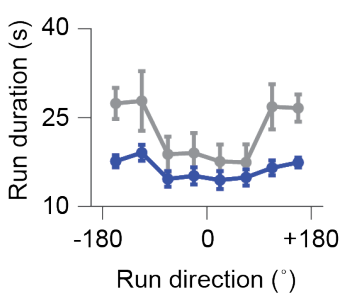

D

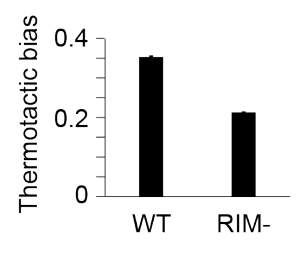

$\mathbf{F}$

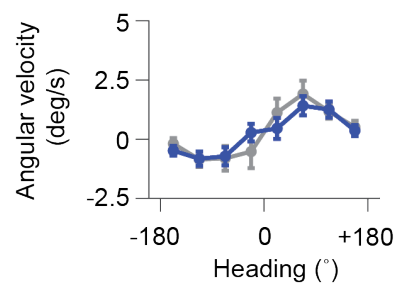

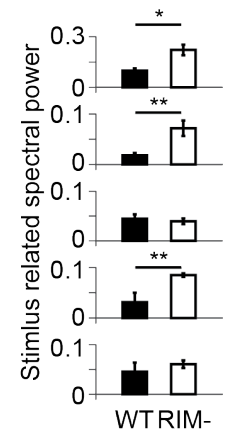

Figure 6. Loss of RIM leads to defects in positive thermotaxis by eliminating CD to AIY. A. Simultaneous measurement of neural activity in AIY and neurons of the motor circuit (AVA, RME, SMDD/V) in RIM ablated animals under oscillating temperature. Sample traces are shown to the left. The distribution and power spectrum of the calcium activity of individual neurons are shown to the right. Gray bands denote the stimulus frequency. B. Comparison of stimulus-related power spectral between wild type and RIM ablated animals $(\mathrm{N}=8$ for wild type, $\mathrm{N}=6$ for RIM ablated animals). Error bars are s.e.m. C. Example trajectories of RIM ablated animals ( $\mathrm{N}=39$ ) cultivated at $25^{\circ} \mathrm{C}$ and exposed to the same thermal gradient as in Figure 1. Top, schematics of the thermal gradient. Middle, trajectories of individual animals during positive thermotaxis. Starting points of all trajectories are aligned (yellow dot); the end points are marked by magenta dots. Bottom, a histogram of the final location of animals at the end of the 20-minute period. D. Averaged thermotactic bias of wild type $(N=140)$ and RIM ablated animals ( $N=102)$. E. Forward run duration as a function of forward run direction in RIM ablated animals (blue) and wild type animals (gray) during thermotaxis. Error bars are $95 \% \mathrm{Cl}$. n.s., non-significant, $*, p<0.05, * *, p<0.01, * * *, p<0.001$ by Wilcoxon rank-sum test. F. Instantaneous angular velocity as a function of the instantaneous heading direction in RIM ablated animals (blue) and wild type animals (gray) during thermotaxis. $(\mathrm{N}=102)$. 
A

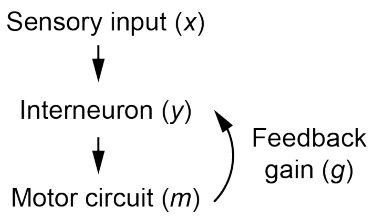

$$
\downarrow
$$

Locomotion

C

D

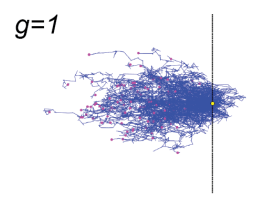

$g=0$
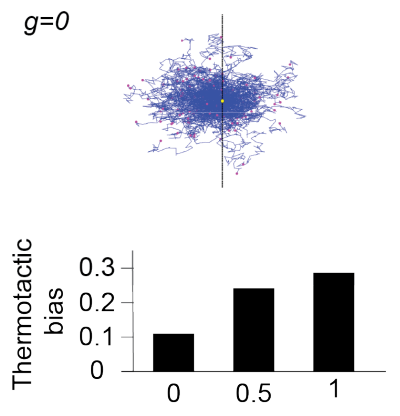

E

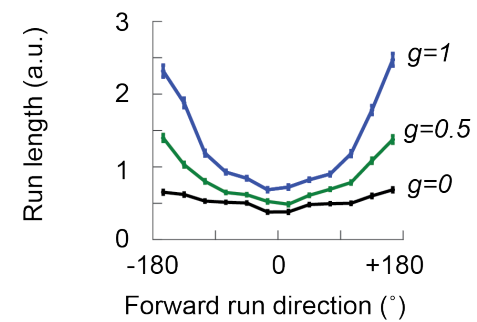

B
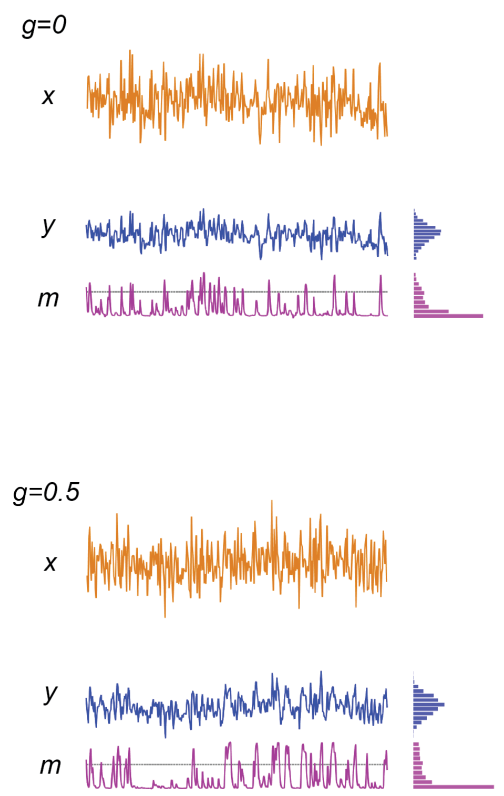

$g=1$

$x$
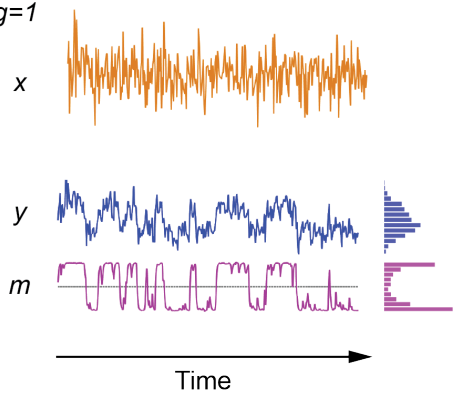

Figure 7. A reduced model explains the role of $C D$ in sustaining forward locomotion during

thermotaxis. A. Schematics of the circuit model. B. Dynamics of the model in response to white noise inputs. Top, temporal profile of the input signal. Middle, dynamics of the model with the strength of CD set to 1. Bottom, dynamics of the model with the strength of CD set to 0 . C. Simulated trajectories of navigational behavior on a 2-D arena with linear input gradient with CD strength set to 0 or 1 . D. Thermotactic biases of simulations with different CD strengths. E. Forward run duration as a function of forward run direction for simulated trajectories 


\section{Acknowledgements}

We thank Daniel Witvliet for insights on C. elegans connectomes and generating Figures $2 \mathrm{~B}$ and 4A. We thank the laboratories of Yun Zhang, Daniel Colón-Ramos, and Cori Bargmann for strains. This work was suppported by NIH P01 GM103770 (ADTS), NIH R01 NS082525-01A1 (ADTS, MZ, MJA), NIH R01 GM084491 (MJA), the Burroughs Wellcome (VV), and the CIHR foundation 154274 (MZ). We thank Steven Flavell and members of the Samuel, Alkema, and Zhen laboratories for constructive advice and help in completion of the study and manuscript preparation.

\section{References}

Aivazian D, Serrano RL, Pfeffer S. TIP47 is a key effector for Rab9 localization. The Journal of Cell Biology. 2006; 173(6):917-926. http://jcb.rupress.org/content/173/6/917, doi: http://dx.doi.org/10.1083/jcb.200510010.

Aksay E, Olasagasti I, Mensh BD, Baker R, Goldman MS, Tank DW. Functional dissection of circuitry in a neural integrator. Nature neuroscience. 2007 apr; 10(4):494-504.

Alkema MJ, Hunter-Ensor M, Ringstad N, Horvitz HR. Tyramine functions independently of octopamine in the Caenorhabditis elegans nervous system. Neuron. 2005 apr; 46(2):247-260. doi: 10.1016/j.neuron.2005.02.024.

Bidaye SS, Machacek C, Wu Y, Dickson BJ. Neuronal Control of Drosophila. Science. 2014; 344(April):97-101. doi: 10.1126/science.1249964.

Bloss CS, Wineinger NE, Peters M, Boeldt DL, Ariniello L, Kim JY, Sheard J, Komatireddy R, Barrett P, Topol EJ. A prospective randomized trial examining health care utilization in individuals using multiple smartphone-enabled biosensors. bioRxiv. 2016; http://biorxiv.org/content/early/2016/01/14/029983, doi: http://dx.doi.org/10.1101/029983.

Brettar I, Christen R, Höfle MG. Aquiflexum balticum gen. nov., sp. nov., a novel marine bacterium of the Cytophaga-Flavobacterium-Bacteroides group isolated from surface water of the central Baltic Sea. International Journal of Systematic and Evolutionary Microbiology. 2004; 54(6):2335-2341. http: //ijs.microbiologyresearch.org/content/journal/ijsem/10.1099/ijs.0.63255-0.

Brettar I, Christen R, Höfle MG. Belliella baltica gen. nov., sp. nov., a novel marine bacterium of the Cytophaga-Flavobacterium-Bacteroides group isolated from surface water of the central Baltic Sea. International Journal of Systematic and Evolutionary Microbiology. 2004; 54(1):65-70. http://ijs. microbiologyresearch. org/content/journal/ijsem/10.1099/ijs.0.02752-0.

Chalasani SH, Chronis N, Tsunozaki M, Gray JM, Ramot D, Goodman MB, Bargmann Cl. Dissecting a circuit for olfactory behaviour in Caenorhabditis elegans Gene-specific control of inflammation by TLR-induced chromatin modifications. Nature. 2008 nov; 451(January):6540-6540. doi: 10.1038/nature06540.

Chalfie M, Sulston JE, White JG, Southgate E, Thomson JN, Brenner S. The neural circuit for touch sensitivity in Caenorhabditis elegans. Journal of Neuroscience. 1985 apr; 5(4):956-964. doi: 10.1523/jneurosci.05-04. 00956.1985.

Chen TW, Wardill TJ, Sun Y, Pulver SR, Renninger SL, Baohan A, Schreiter ER, Kerr RA, Orger MB, Jayaraman V, Looger LL, Svoboda K, Kim DS. Ultrasensitive fluorescent proteins for imaging neuronal activity. Nature. 2013; 499(7458):295-300. doi: 10.1038/nature12354.

Clark DA, Biron D, Sengupta P, Samuel ADT, Clark, Biron D, Sengupta P, Samuel ADT. The AFD sensory neurons encode multiple functions underlying thermotactic behavior in Caenorhabditis elegans. Journal of Neuroscience. 2006; 26(28):7444-7451. doi: 10.1523/jneurosci.1137-06.2006.

Clark DA, Clark DA, Gabel CV, Gabel CV, Gabel H, Gabel H, Samuel ADT. Temporal Activity Patterns in Thermosensory Neurons of Freely Moving Caenorhabditis elegans Encode Spatial Thermal Gradients. Journal of Neuroscience. 2007 jun; 27(23):6083-6090. http://www.jneurosci.org/content/jneuro/27/23/6083.full.pdfhttp://www. ncbi.nlm.nih.gov/pubmed/17553981 http://www.jneurosci.org/cgi/doi/10.1523/JNEUROSCI.1032-07.2007, doi: 10.1523/JNEUROSCI.1032-07.2007.

Ferrée TC, Ferrée F, Lockery SR. Computational Rules for Chemotaxis in the Nematode C. elegans; 1999.

Fu Y, Tucciarone JM, Espinosa JS, Sheng N, Darcy DP, Nicoll RA, Huang ZJ, Stryker MP. A cortical circuit for gain control by behavioral state. Cell. 2014 mar; 156(6):1139-1152. doi: 10.1016/j.cell.2014.01.050. 
Gao S, Xie L, Kawano T, Po MD, Pirri JK, Guan S, Alkema MJ, Zhen M. The NCA sodium leak channel is required for persistent motor circuit activity that sustains locomotion. Nature communications. 2015 feb; 6:ncomms7323.

Gordus A, Pokala N, Levy S, Flavell SW, Bargmann Cl. Feedback from Network States Generates Variability in a Probabilistic Olfactory Circuit. Cell. 2015 mar; .

Gray JM, Hill JJ, Bargmann Cl. A circuit for navigation in Caenorhabditis elegans. Proceedings of the National Academy of Sciences of the United States of America. 2005 mar; 102(9):3184-3191. doi: 10.1073/pnas.0409009101.

Hawk JD, Calvo AC, Liu P, Almoril-Porras A, Aljobeh A, Torruella-Suárez ML, Ren I, Cook N, Greenwood J, Luo L, Wang ZWW, Samuel ADT, Colón-Ramos DA. Integration of Plasticity Mechanisms within a Single Sensory Neuron of C. elegans Actuates a Memory. Neuron. 2018 jan; 97(2):356-367.e4. http://linkinghub.elsevier. com/retrieve/pii/S0896627317311741, doi: 10.1016/j.neuron.2017.12.027.

Hedgecock EM, Russell RL. Normal and mutant thermotaxis in the nematode Caenorhabditis elegans. Proceedings of the National Academy of Sciences of the United States of America. 1975 oct; 72(10):4061-4065. doi: 10.1073/pnas.72.10.4061.

Hendricks M. Compartmentalized calcium dynamics in a C. elegans interneuron encode head movement. Nature. 2012 jul; 487(7405):99-103. doi: 10.1038/nature11081.

Herberholz J, Antonsen BL, Edwards DH. A lateral excitatory network in the escape circuit of crayfish. The Journal of Neuroscience. 2002 oct; 22(20):9078-9085.

Hobert O, Mori I, Yamashita Y, Honda H, Ohshima Y, Liu Y, Ruvkun G. Regulation of interneuron function in the C. elegans thermoregulatory pathway by the ttx-3 LIM homeobox gene. Neuron. 1997 aug; 19(2):345-357. doi: 10.1016/S0896-6273(00)80944-7.

Hoopfer ED, Jung Y, Inagaki HK, Rubin GM, Anderson DJ. P1 interneurons promote a persistent internal state that enhances inter-male aggression in Drosophila. eLife. 2015; 4(DECEMBER2015):1-27. doi: 10.7554/eLife.11346.

Ino Y, Yoshida K. Parallel use of two behavioral mechanisms for chemotaxis in Caenorhabditis elegans. Journal of Neuroscience. 2009 apr; 29(17):5370-5380. doi: 10.1523/JNEUROSCI.3633-08.2009.

Kato S, Kaplan HS, Schrödel T, Skora S, Lindsay TH, Yemini E, Lockery S, Zimmer M. Global Brain Dynamics Embed the Motor Command Sequence of Caenorhabditis elegans. Cell. 2015 oct; 163(3):656-669. http://www. ncbi.nlm.nih.gov/pubmed/26478179http://linkinghub.elsevier.com/retrieve/pii/S0092867415011964, doi: 10.1016/j.cell.2015.09.034.

Kawano T, Po MD, Gao S, Leung G, Ryu WS, Zhen M. An imbalancing act: Gap junctions reduce the backward motor circuit activity to bias C. elegans for forward locomotion. Neuron. 2011 nov; 72(4):572-586. http: //dx.doi.org/10.1016/j.neuron.2011.09.005, doi: 10.1016/j.neuron.2011.09.005.

Klapoetke NC, Murata Y, Kim SS, Pulver SR, Birdsey-Benson A, Cho YK, Morimoto TK, Chuong AS, Carpenter EJ, Tian Z, Wang J, Xie Y, Yan Z, Zhang Y, Chow BY, Surek B, Melkonian M, Jayaraman V, Constantine-Paton M, Wong GKS, et al. Independent optical excitation of distinct neural populations. Nature Methods. 2014 mar; 11(3):338-346.

Kocabas A, Shen CH, Guo ZV, Ramanathan S. Controlling interneuron activity in Caenorhabditis elegans to evoke chemotactic behaviour. Nature. 2012 oct; 490(7419):273-277. doi: 10.1038/nature11431.

Lee SH, Dan Y. Neuromodulation of brain states. Neuron. 2012 oct; 76(1):209-222.

Li WC, Soffe SR, Wolf E, Roberts A. Persistent responses to brief stimuli: feedback excitation among brainstem neurons. The Journal of Neuroscience. 2006 apr; 26(15):4026-4035.

Li Z, Liu J, Zheng M, Xu XZS. Encoding of both analog- and digital-like behavioral outputs by one C. Elegans interneuron. Cell. 2014 nov; 159(4):751-765. doi: 10.1016/j.cell.2014.09.056.

Lim MA, Chitturi J, Laskova V, Meng J, Findeis D, Wiekenberg A, Mulcahy B, Luo L, Li Y, Lu Y, Hung W, Qu Y, Ho CY, Holmyard D, Ji N, McWhirter R, Samuel ADT, Miller DM, Schnabel R, Calarco JA, et al. Neuroendocrine modulation sustains the C. elegans forward motor state. eLife. 2016 nov; 5(NOVEMBER2016). http://elifesciences.org/lookup/doi/10.7554/eLife.19887https://elifesciences.org/content/5/e19887, doi: 10.7554/eLife.19887. 
Luo L, Cook N, Venkatachalam V, Martinez-Velazquez LA, Zhang X, Calvo AC, Hawk J, Maclnnis BL, Frank M, Ng JHR, Klein M, Gershow M, Hammarlund M, Goodman MB, Colon-Ramos DA, Zhang Y, Samuel ADT, ColónRamos DA, Zhang Y, Samuel ADT, et al. Bidirectional thermotaxis in Caenorhabditis elegans is mediated by distinct sensorimotor strategies driven by the AFD thermosensory neurons. Proceedings of the National Academy of Sciences. 2014 feb; 111(7):2776-2781. http://www.pnas.org/cgi/doi/10.1073/pnas.1315205111, doi: 10.1073/pnas.1315205111.

Luo L, Wen Q, Ren J, Hendricks M, Gershow M, Qin Y, Greenwood J, Soucy ER, Klein M, Smith-Parker HK, Calvo AC, Colón-Ramos DA, Samuel ADT, Zhang Y, Colon-Ramos D, Samuel ADT, Zhang Y, Colón-Ramos DA, Samuel ADT, Zhang Y, et al. Dynamic encoding of perception, memory, and movement in a C. elegans chemotaxis circuit. Neuron. 2014; 82(5):1115-1128. doi: 10.1016/j.neuron.2014.05.010.

Major G, Tank D. Persistent neural activity: prevalence and mechanisms. Current opinion in neurobiology. 2004 dec; 14(6):675-684.

McCormick KE, Gaertner BE, Sottile M, Phillips PC, Lockery SR. Microfluidic Devices for Analysis of Spatial Orientation Behaviors in Semi-Restrained Caenorhabditis elegans. PloS one. 2011 oct; 6(10):e25710.

McQuilton P, St Pierre SE, Thurmond J, the FlyBase Consortium. FlyBase 101 - the basics of navigating FlyBase. Nucleic Acids Research. 2012; 40(D1):D706-D714. http://nar.oxfordjournals.org/content/40/D1/D706.abstract, doi: http://dx.doi.org/10.1093/nar/gkr1030.

Mori I, Ohshima Y. Neural regulation of thermotaxis in Caenorhabditis elegans. Nature. 1995 jul; $376(6538): 344-$ 348. doi: $10.1038 / 376344 a 0$.

Narayan A, Laurent G, Sternberg PW. Transfer characteristics of a thermosensory synapse in Caenorhabditis elegans. Proceedings of the National Academy of Sciences of the United States of America. 2011 jun; 108(23):9667-9672. doi: 10.1073/pnas.1106617108.

Ouellette MH, Desrochers MJ, Gheta I, Ramos R, Hendricks M. A Gate-and-Switch Model for Head Orientation Behaviors in Caenorhabditis elegans. Eneuro. 2018; 5(6):ENEURO.0121-18.2018. doi: 10.1523/eneuro.0121. 18.2018.

Petreanu L, Mao T, Sternson SM, Svoboda K. The subcellular organization of neocortical excitatory connections. Nature. 2009 jan; 457(7233):1142-1145. doi: 10.1038/nature07709.

Pfister R, Schwarz K, Janczyk M, Dale R, Freeman J. Good things peak in pairs: a note on the bimodality coefficient. Frontiers in Psychology. 2013; 4:700. https://www.frontiersin.org/article/10.3389/fpsyg.2013.00700, doi: 10.3389/fpsyg.2013.00700.

Pierce-Shimomura JT, Morse TM, Lockery SR, Lockery SR. The fundamental role of pirouettes in Caenorhabditis elegans chemotaxis. The Journal of Neuroscience. 1999 nov; 19(21):9557-9569.

Qi YB, Garren EJ, Shu X, Tsien RY, Jin Y. Photo-inducible cell ablation in Caenorhabditis elegans using the genetically encoded singlet oxygen generating protein miniSOG. Proceedings of the National Academy of Sciences of the United States of America. 2012 may; 109(19):7499-7504. doi: 10.1073/pnas.1204096109.

Ryu WS, Samuel ADT. Thermotaxis in Caenorhabditis elegans analyzed by measuring responses to defined Thermal stimuli. The Journal of Neuroscience. 2002 jul; 22(13):5727-5733.

Schneider DM, Nelson A, Mooney R. A synaptic and circuit basis for corollary discharge in the auditory cortex. Nature. 2014 sep; 513(7517):189-194. doi: 10.1038/nature13724.

Seelig JD, Jayaraman V. Neural dynamics for landmark orientation and angular path integration. Nature. 2015 may; 521(7551):186-191. doi: 10.1038/nature14446.

Serrano-Saiz E, Poole RJ, Felton T, Zhang F, De La Cruz ED, Hobert O. XModular control of glutamatergic neuronal identity in C. elegans by distinct homeodomain proteins. Cell. 2013 oct; 155(3):659. doi: 10.1016/j.cell.2013.09.052.

Seung HS. How the brain keeps the eyes still. Proceedings of the National Academy of Sciences. 1996 nov; 93(23):13339-13344.

Shen Y, Wen Q, Liu H, Zhong C, Qin Y, Harris G, Kawano T, Wu M, Xu T, Samuel ADT, Zhang Y. An extrasynaptic GABAergic signal modulates a pattern of forward movement in Caenorhabditis elegans. eLife. 2016; 5(MAY2016):e14197. http://dx.doi.org/10.7554/eLife.14197, doi: 10.7554/eLife.14197. 
Shu X, Lev-Ram V, Deerinck TJ, Qi Y, Ramko EB, Davidson MW, Jin Y, Ellisman MH, Tsien RY. A Genetically Encoded Tag for Correlated Light and Electron Microscopy of Intact Cells, Tissues, and Organisms. PLoS biology. 2011 apr; 9(4):e1001041.

Tsalik EL, Hobert O. Functional mapping of neurons that control locomotory behavior in Caenorhabditis elegans. Journal of Neurobiology. 2003 jun; 56(2):178-197. doi: 10.1002/neu.10245.

Wakabayashi T, Kitagawa I, Shingai R. Neurons regulating the duration of forward locomotion in Caenorhabditis elegans. Neuroscience Research. 2004 sep; 50(1):103-111. doi: 10.1016/j.neures.2004.06.005.

Ward S. Chemotaxis by the nematode Caenorhabditis elegans: identification of attractants and analysis of the response by use of mutants. Proceedings of the National Academy of Sciences of the United States of America. 1973 mar; 70(3):817-821. doi: 10.1073/pnas.70.3.817.

White JG, Southgate E, Thomson JN, Brenner S. The Structure of the Nervous System of the Nematode Caenorhabditis elegans. Philosophical Transactions of the Royal Society B: Biological Sciences. 1986; 314(1165):1-340. doi: 10.1098/rstb.1986.0056.

Yamaguchi S, Naoki H, Ikeda M, Tsukada Y, Nakano S, Mori I, Ishii S. Identification of animal behavioral strategies by inverse reinforcement learning. PLoS Computational Biology. 2018; 14(5):1-20. http://dx.doi.org/10.1371/ journal.pcbi.1006122, doi: 10.1371/journal.pcbi.1006122.

Zagha E, Casale AE, Sachdev RNS, McGinley MJ, McCormick DA. Motor cortex feedback influences sensory processing by modulating network state. Neuron. 2013 aug; 79(3):567-578. doi: 10.1016/j.neuron.2013.06.008.

Zhang Y, Lu H, Bargmann Cl. Pathogenic bacteria induce aversive olfactory learning in Caenorhabditis elegans. . 2005 nov; 438(7065):179-184. 
620 Appendix 1

621

\section{Constructs and transgenic arrays}

\section{Calcium imaging}

Injection Marker

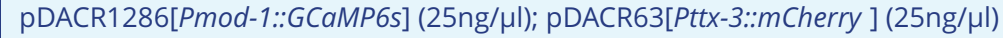

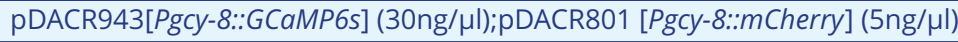

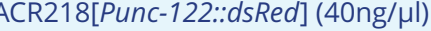

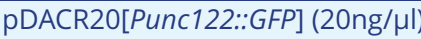

Transgene

train

pJH3338[Pg/r-1-GCaMP6s::wCherry]

pL15EKlin-15AB genomic DNA ( 20ng/ $\mu$ )

aeals003(A/Y) (integrated olaEx1621

aeals004 (AFD) (integrated olaEx1527

hpls471 (premotor/ motor)

ZM8558

\section{Optogenetic stimulation}

pHR2[Plgc-55B-Chrimson::wCherry]

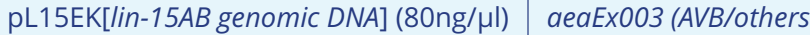

ADS029

pHR6[Prig-3-Chrimson::wCherry]

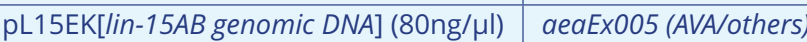

ADS031

\section{Cell ablation}

pJH2829[Pcex-1- MiniSOG::SL2::wCherry]

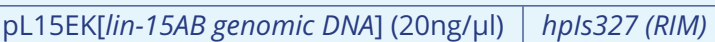

ZM7978

pJH3311[Pinx-1- MiniSOG::SL2::wCherry]

pL15EK[lin-15AB genomic DNA] (20ng/ul)

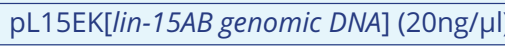

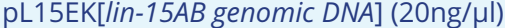

hpls465(AlB)

ZM8484

pJH2931 [Prig-3- MiniSOG::SL2:::wCherry]

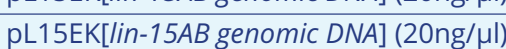

hpEx3072 (AVA/others)

ZM7198

p]H2890[Plgc-55B-MiniSOG::SL2::wCherry]

hpls331(AVB/others)

ZM7297

\section{Synaptic manipulation}

Pttx-3::TeTx::mCherry

yx/s25 (A/Y)

Ptdc-1::TeTx:m

kyEx4962 (RIM/RIC)

${ }^{a}$ Gift of Daniel Colon-Ramos

${ }^{b}$ Gift of Daniel Colon-Ramos

'Zhang et al. (2005)

${ }^{d}$ Gordus et al. (2015) 
${ }_{623}$ Appendix 2

624

Strains

For thermotaxis and locomotion assays

\begin{tabular}{|c|c|c|c|}
\hline Strain & Genotype & Purpose & Figure \\
\hline Bristol N2 & wild-type & Wild-type behavior & Figure 1A-D \\
\hline ZM7978 & hpls327 & Behavior upon RIM ablation & Figure $6 \mathrm{C}-\mathrm{F}$ \\
\hline \multicolumn{4}{|c|}{ Calcium imaging } \\
\hline ADS003 & aeas/s003 & AIY imaging & Figure 2D;3A,B \\
\hline ADS004 & aeals004 & AFD imaging & Figure $2 \mathrm{~A}$ \\
\hline ADS027 & aeals003; hpls471 & Simultaneous imaging of AIY, AVA, RME, SMDD, SMDV and RIM & Figure 2C,D \\
\hline ADS043 & aeals003; yxIs25 & AIY imaging, upon blockade of AIY chemical transmission & Figure 3B \\
\hline ADS010 & aeals003; hpls327 & AIY imaging, upon ablation of RIM & Figure $4 B, C ; 5 A, B, D ; 6 A, B$ \\
\hline ADS014 & aeals003; hpls321 & AIY imaging, upon ablation of RIM, AVA, AVE, AVD and PVC & Figure 4B \\
\hline ADS026 & aeals003; hpEx3072 & AIY imaging upon ablation of AVA & Figure 4B \\
\hline ADS036 & aeals003; hpls331 & AlY imaging, upon ablation of AVB & Figure 4B \\
\hline ADS046 & aeals003; hpls465 & AIY imaging, upon ablation of AIB & Figure 4B,D \\
\hline ADS029 & aeaEx003; aeals003; lite-1(ce314) & AIY imaging upon optogenetic stimulation of AVB & Figure 4E \\
\hline ADS031 & aeaEx005; aeals003; lite-1(ce314) & AIY imaging upon optogenetic stimulation of AVA & Figure 4E \\
\hline ADS033 & aeaEx005; aeals003; hpls327; lite-1(ce314) & AIY imaging, upon RIM ablation and AVA stimulation & Figure 4E \\
\hline ADS035 & aeaEx003; aeals003; hpls327; lite-1(ce314) & AIY imaging, upon RIM ablation and AVB stimulation & Figure 4E \\
\hline ADS013 & aeals003; kyEx4962 & AIY imaging, upon disruption of RIM/RIC chemical transmission & Figure 5C,D \\
\hline ADS006 & aeals003;tdc-1(n3419) & AIY imaging in tyramine/octopamine synthesis mutant & Figure 5C,D \\
\hline QW1411 & aeals003; eat-4(ky5) & AIY imaging in glutamate mutant & Figure 5C,D \\
\hline QW1175 & aeals003; unc-31(e928) & AIY imaging in dense core vesicle release mutant & Figure 5C,D \\
\hline QW1408 & aeals003; cat-1(e1111) & AIY imaging in biogenic amine transporter mutant & Figure 5C,D \\
\hline
\end{tabular}


moving animals

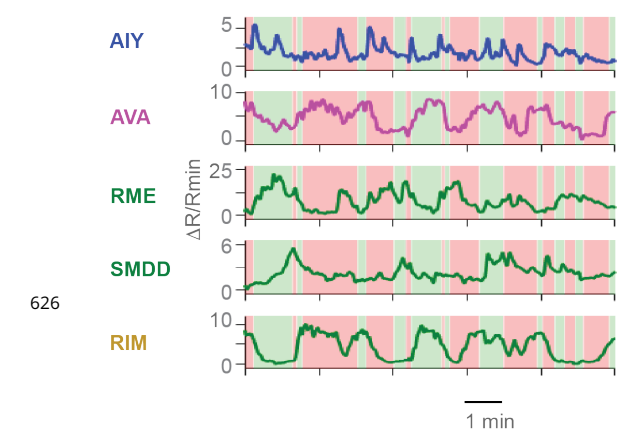

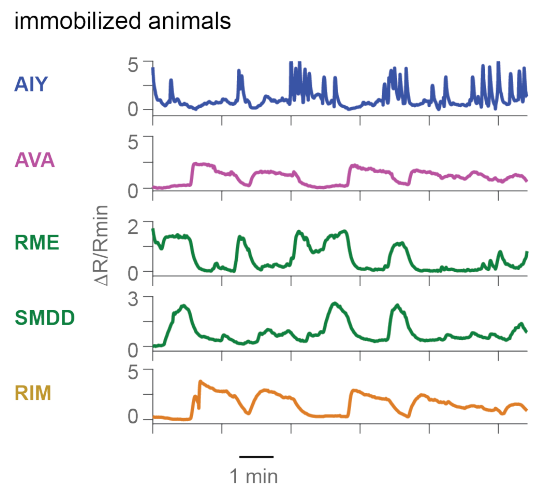

Figure 2-Figure supplement 1. Analysis of neural activity in the thermotaxis circuit in immobilized and moving animals without temperature stimulation.A. Simultaneous measurement of calcium transient changes of neurons labeled by the transgenic reporter in moving (left) and immobilized (right) animals at constant temperature $\left(T=15^{\circ} \mathrm{C}\right)$. B. Cross correlation between the activity of RIM and that of other neurons measured in (A) in immobilized and moving animals. 